\title{
Extra Glycemic Impacts of GLP-1 Receptor Agonists: Benefits of a Class Effect?
}

\author{
Lis Marina de Mesquita Araújo1, Sumaya Feguri1, Thereza Lopes de Oliveira1, \\ Fernanda Batista Pedrosa1, Rodrigo Garcez Guimarães ${ }^{1}$, \\ Larissa Bianca Paiva Cunha de Sá1, Denise Rosso Tenório Wanderley Rocha ${ }^{1}$, \\ Alberto Krayyem Arbex ${ }^{1,2}$ \\ ${ }^{1}$ Division of Endocrinology, IPEMED Medical School, Belo Horizonte, Brazil \\ ${ }^{2}$ Harvard T. H. Chan School of Public Health, Boston, USA \\ Email: lismarina@hotmail.com
}

Received 13 December 2015; accepted 18 January 2016; published 21 January 2016

Copyright (C) 2016 by authors and Scientific Research Publishing Inc.

This work is licensed under the Creative Commons Attribution International License (CC BY). http://creativecommons.org/licenses/by/4.0/

c) (i) Open Access

\begin{abstract}
GLP-1 receptor agonists are approved for the treatment of type 2 diabetes, and more recently for obesity treatment. The glucagon-like-peptide-1 (GLP-1) is a glucose dependent hormone produced by intestinal cells, which is involved in insulin secretion and glucagon suppression. This hormone controls glucose plasma levels and reduces food intake. Additional effects were reported in slowing gastric emptying and in inducing satiety. In clinical practice, the GLP-1 receptor agonists are associated with significant reductions in glycosylated hemoglobin (HbA1c) and weight loss, despite showing a low risk of hypoglycemia. Beneficial effects have also been observed on blood pressure and lipid profile. The most common side effects associated with GLP-1 receptor agonists are gastrointestinal motility disorders, such as nausea, vomiting and diarrhea, which are not associated with long-term health risks. Therefore, GLP-1 receptor agonists represent a relevant medication for type 2 diabetes, whose benefits may go far beyond glycemic control.
\end{abstract}

\section{Keywords}

Diabetes, GLP-1 Receptor Agonists, Glycemic Control, Extra Glicemic Effects, Weight Loss, Cardiovascular Risk

\section{Introduction}

The glucagon-like peptide 1 (GLP-1) is an incretin hormone secreted mainly by the enteroendocrine cells of the intestine in response to glucose. Native GLP-1 has a short half-life of minutes, being rapidly degraded by dipep- 
tidyl peptidase-IV (DPP-IV), to generate an NH2-terminally truncated metabolite in addition to undergoing renal excretion [1]. The GLP-1 receptor is widely expressed in islet cells, kidney, lung, brain, the gastrointestinal tract, and, interestingly, also in the heart [2].

The GLP-1 is also an insulinotropic agent through its ability to stimulate insulin gene expression and proinsulin biosynthesis [3] and acts as a potent-cell growth factor [4] and also increases the expression level of the cell specific transcription factor - the pancreatic duodenal homeobox gene 1 (PDX-1) [4] [5]. In addition, the glucose dependent mechanism increases beta cells proliferation in a phosphatidylinositol-3 kinase (PI-3K) dependent way in (INS-1) cells [4] as well as increased pancreatic islet mass in mouse pancreas [5].

Therapies that act on incretin system involve two main hormones: glucagon as incretin peptide-1 (GLP-1) and glucose-dependent insulinotropic polypeptide (GIP), which are quickly released after meals [6]. Both hormones are glucose-dependent insulin secretion; and GLP-1, but not GIP, also suppresses glucagon secretion, delays gastric emptying, and decreases food intake. GLP-1 and GIP are inactivated by DPP-4 [6].

\section{Types of Incretin Based Therapies}

This therapy consists of two classes of drugs: GLP-1 receptor agonists, which have biological activity similar to GLP-1, but are resistant to DPP-IV; and DPP-4 inhibitors, which prevent enzymatic inactivation of endogenous GLP-1 and GIP [7]. Both classes increase insulin and suppress glucagon secretions in a glucose-dependent way, but with differences. Glycogenic benefits of native GLP-1 or a GLP-1 receptor agonist (dosed to pharmacological concentrations) are accompanied by delayed gastric emptying and weight loss, but nausea initially occurs in few people [6] [8].

\section{Types of Glucagon-Like Peptide-1 Analogues}

\section{1) Liraglutide}

Liraglutide (approved by EU, Japan, 2009, USFDA, 2010; marketed by NovoNordisk, brand name Victoza). Liraglutide is a human GLP-1 analogue with one amino acid substitution (Arg34Lys) and a C-16 palmitic acid side chain attached via a glutamyl spacer. These modifications result in slower absorption from subcutaneous tissue, reversible albumin binding, and resistance to GLP-1 inactivation by dipeptidyl peptidase-4. Liraglutide is 99\% bound to albumin, with free liraglutide degraded by endogenous peptidases, and not via renal elimination [9]. Liraglutide injection produces maximal concentrations within 10 - $14 \mathrm{~h}$ after administration, with a half-life of $13 \mathrm{~h}$ [10]. This drug has been developed as a treatment for T2DM, as an adjunct to lifestyle therapy and could be associated with others oral antidiabetic drugs [11].

\section{2) Exenatide}

Exenatide (approved by the FDA, 2005; available for use marketed by Eli Lilly; brand name Byetta). Exanatide (exendin-4) is a 39-amino acid peptide incretin mimetic that exhibits glucoregulatory activities similar to the mammalian incretin hormone glucagon-like peptide 1 (GLP-1) [12] [13]. Exenatide's glucose-dependent enhancement of insulin secretion may be mediated by exenatide binding to the pancreatic GLP-1 receptor [14]. In animal models of diabetes and in insulin-secreting cell lines, exenatide and GLP-1 reportedly improve $\beta$-cell function by increasing the expression of key genes involved in insulin secretion, increasing insulin biosynthesis, and augmenting $\beta$-cell mass through multiple mechanisms [15]. Data obtained in animal models also indicate that exenatide and GLP-1 reduce food intake, cause weight loss, and have an insulin-sensitizing effect [15]-[19].

3) Exenatide LAR (sustained release; once weekly)

Exenatide LAR (approved by EU, Japan, 2009, USFDA, 2010; marketed by AstraZeneca, brand name Bydureon). Exenatide once-weekly (EQW [2 $\mathrm{mg}$ s.c.]) is a long-acting formulation contains the active ingredient of the original exenatide twice-daily (EBID) formulation encapsulated in 0.06-mm-diameter microspheres of medical-grade poly-(d,l-lactide-co-glycolide) (PLG). After mechanical suspension and subcutaneous injection by the patient, EQW microspheres hydrate in situ and adhere to one another to form an amalgam [20]. EQW provides constant exposure to Exenatide following once-weekly, patient-administered, subcutaneous injections. Like EBID, EQW has been shown to reduce HbA1c, improve fasting glucose, and reduce body weight [21]-[23].

Advantages of EBID over EQW include modestly better postprandial control for the two post-injection meals and fewer injection site adverse events. Advantages of EQW over EBID include a greater ability to meet therapeutic HbA1c goals, better fasting glucose control, a lower incidence of gastrointestinal upset, more flexible dosing, and less frequent injections (one vs. 14 per week) [20]. 


\section{4) Dulaglutide (sustained release; once weekly)}

Dulaglutide it is a recent long-acting GLP-1 analogue (approved by the FDA on 18 September 2015; marketed by Eli Lilly \& Co.; brand name Trulicity) projected to have a convenient once a week dosing. In a randomized placebo-controlled double-blinded study (262 obese type 2 patients), an HbA1c reduction of approximately $-1.28 \%-1.52 \%$ was found, along with weight loss of -1.40 and $-2.51 \mathrm{~kg}$. Upper gastrointestinal symptoms of nausea (13\%), diarrhea (9\%), and abdominal distension (8\%) were the most frequently reported adverse events [24] [25]. Dulaglutide was approved with similar concerns of other GLP-1R agonists by the FDA, such as a post-marketing cardiovascular risk trial, other trials to evaluate risks of thyroid carcinoma and safety use among children, along with analyses regarding kidney safety and SNC safety of use.

The main advantage of Dulaglutide is its posology: a once-weekly subcutaneous injection to improve glycemic control, with promising prospects for adherence and possible similar results to other drugs of its class.

\section{5) Lixisenatide}

Lixisenatide is a new synthetic GLP-1 receptor agonist with extended biological activity that is currently being developed for the treatment of patients with T2DM (approved in Europe in 2013, accepted for filing the New Drug Application by the FDA on October 2015; marketed by Sanofi; brand name Lyxumia). It is a 44 amino acid peptide that is amidated at the C-terminal amino acid, and shares some structural elements with exendin-4 [26].

Lixisenatide protects $\beta$-cells derived from a rat pancreatic cell line from lipid and cytokine-induced apoptosis. It also prevents lipotoxic islet insulin depletion and preserves insulin production, storage and pancreatic $\beta$-cell function in human islets [27]. Furthermore, Lixisenatide enhances insulin biosynthesis and stimulates $\beta$-cell proliferation in animal models, suggesting the potential to modify the progression of diabetes. The enhancement of glucose-stimulated insulin secretion (GSIS) provided by Lixisenatide occurs in a strictly glucose-dependent way, and thus has the potential to avoid undesirable hypoglycaemia. In animal models of diabetes, Lixisenatide improves basal blood glucose and HbA1c with a rapid onset and sustained duration of action, and prevents the deterioration of pancreatic responsiveness and glucose homeostasis over time. Lixisenatide also delays gastric emptying and reduces food intake, which may be important for both its glucose-lowering effect and for the induction of weight loss [28]. Thus, based on its preclinical pharmacological profile, Lixisenatide shows actions that suggest it may be a promising therapy for T2DM.

\section{6) Albiglutide}

Albiglutide (approved by the FDA on April 2014; marketed by GlaxoSmithKline; brand names Eperzan(Europe) and Tanzeum(US)) is a GLP-1 receptor agonist developed through the fusion of two repeats of human GLP-1 molecules to recombinant human albumin [29]. Albiglutide is relatively impermeant to the central nervous system [30] which may have implications for GI tolerability. In nonclinical studies, Albiglutide stimulated cAMP production through the GLP-1 receptor and induced insulin secretion from INS-1 cells in vitro and in animal models [30] [31]. It also delayed gastric emptying and reduced food intake in rodents [30] [32].

In conclusion, Albiglutide improved glucose control in a dose-dependent way when given weekly and biweekly. Higher monthly doses of Albiglutide were efficacious, but their use was constrained by the higher frequency of GI-related adverse events. Weekly Albiglutide significantly improved glycemic control with an acceptable safety and tolerability profile and modest weight loss without increasing the risk of hypoglycemia or immunological response in subjects with type 2 diabetes. Future studies may elucidate whether titration dosing or biweekly scheduling could be options for patients who respond to and tolerate the initial weekly regimen [33].

\section{Glycemic Efficacy of GLP-1 Receptor Agonists}

Peptide-1 receptor agonists similar to glucagon (GLP-1 RAs) are available for the treatment of type 2 diabetes mellitus (T2DM) for nearly 10 years now, and show extensive clinical experience. Several drugs are marketed, including exenatide, exenatide long release (LAR), liraglutide, lixisenatide, albiglutide and dulaglutide, while others are under development [34] [35].

Exenatide, exenatide LAR, and lixisenatide are synthetic peptides based on exendin-4, which is found in the saliva of the Gila monster lizard. Exendin-4 has homology of 53\% to the human GLP-1 with equal power in the GLP-1 receptor and is resistant to degradation by serine protease enzyme dipeptidyl peptidase-4 (DPP-4) [36]. Other GLP-1 RAs synthetic peptides are modified GLP-1 naturally occurring, where the amino acid substitution 
has been used to protect the GLP-1 molecule to be broken by DPP-4 [35].

All GLP-1 RAs are administered subcutaneously and are effective in improving glycemic control (as measured at the level of glycated hemoglobin [HbA1c]) [35] [37] [38]. Glycemic control with GLP-1 RAs is achieved by their ability to stimulate insulin secretion, inhibiting glucagon release (so glucose-dependent), decreasing gastric emptying, and increased satiety for people eat less. Ideally, the treatment of T2DM reduced HbA1c levels for a person without diabetes, but this must be balanced against the risk of inducing side effects, particularly hypoglycemia [37] [38]. Due to their glucose-dependent action, GLP-1 RAs are associated with a low risk of hypoglycemia. For most individuals with T2DM, the glycemic targets set by the American Diabetes Association/European Association for the Study of Diabetes and the American Association of Clinical Endocrinologists are less than $53 \mathrm{mmol} / \mathrm{mol}(7.0 \%)$ and less than $47 \mathrm{mmol} / \mathrm{mole}$ (6.5\%) respectively [37] [38]. It is estimated that for a reduction of $11 \mathrm{mmol} / \mathrm{mol}$ (1\%) in HbA1c reduce cardiovascular complications by approximately $40 \%$ [39]. The pharmacological doses of GLP-1 RAs generally result in higher glycemic control compared to other medications for diabetes, including the DPP-4 inhibitors and sulfonylureas. They are also associated with other advantages, particularly the ability to induce weight loss [40] [41].

GLP-1 RAs can be categorized into short or long duration agents. Short agents (exenatide and lixisenatide) have half-lives of 2 - 3 hours with exenatide administered twice daily and once daily lixisenatide. They decrease in HbA1c levels and a similar moderate glycemic efficacy $(5.5 \mathrm{mmol} / \mathrm{mol}[0.5 \%] 10 \mathrm{mmol} / \mathrm{mol}$ [0.9\%]). The fasting plasma glucose (FPG), and postprandial glucose (PPG) high contribute to high levels of HbA1c [27]. Short-acting compounds have pronounced effects on the PPG due, in part, to inhibition of gastric emptying. The lixisenatide shown to have sustained effects on gastric emptying [29] [30]. It is remarkable decreases of postprandial insulin after GLP-1 administration ARs short-acting because the delayed gastric emptying markedly reduces postprandial glucose response [42].

The long term compounds are administered once daily (liraglutide) or once a week (exenatide LAR, albiglutide, dulaglutide). The long half-life of these compounds is due to the formulation (exenatide LAR) or to the conjugation with various other molecules such as the acyl group (liraglutide), human albumin (albiglutide), or Fc fusion protein with immunoglobulin G (dulaglutide), which increase their size, affecting their pharmacokinetics, efficacy, and safety. The longer half-life allows continuous activation of the GLP-1 receptor and less fluctuations in peptide levels [41]. In general, liraglutide is associated with increased efficacy compared to exenatide. On average, liraglutide decreases HbA1c in about $4 \mathrm{mmol} / \mathrm{mol}(0.33 \%)$ and FPG at $1 \mathrm{mmol} / \mathrm{L}$ more than exenatide [34] [40] [43]-[46]. The other compounds of longer acting, once a week, in general, showed less potent reductions in HbA1c compared to liraglutide [34] [43] [45]. However, once a week dulaglutide recently proved to be not less than liraglutide once daily [40]. Comparative clinical studies allow a direct comparison of the clinical benefits and safety of medicines with similar mechanisms of action [45]. Most of the GLP-1 RAs has been compared to exenatide or liraglutide, the first GLP-1 RAs and with more clinical experience [45]. Long- term studies showed that the beneficial effects of GLP-1 RAs are maintained for more than 2 years and show superiority over sulfonylureas [41] [47] [48].

\section{The Extra Glycemic Actions of GLP-1 Receptor Agonists}

The GLP-1 receptors (GLP-1R) are found throughout the body, including in the cardiovascular system, therefore providing a larger field to relevant metabolic influences such as diabetes and vascular complications. Specific receptors have been identified for G-protein coupled GLP-1 in tissues of the gastrointestinal tract, pancreas, cardiac myocytes, liver, lung, blood vessels including the endothelium of the coronary artery, macrophages, peripheral nerves and the central nervous system.

In experimental methods of specific mRNA transcriptions of GLP-1R were detected by RT-PCR in heart of rats and in the human heart. Furthermore, immunohistochemical examination detected GLP-1R protein in cardiac myocytes and in the endocardium of rats, while Western Blot revealed GLP-1R in all heart chambers of the rats. In neonatal rat cardiac myocytes in culture, treatment with exenatide $(3 \mathrm{~nm})$ during 20 min increased Akt and Erk, both regulators that characterize the growth of cardiac myocytes and glucose metabolism [49].

GLP-1 RAS slows gastric emptying providing weight loss, but preclinical studies suggest that the major mechanism associated with weight loss promoted by GLP-1 RAS results of the effects on appetite, which promotes by brain signals [50]. These anorexigenic effects of GLP-1 seem to be transmitted by the paraventricular and arcuate nucleus area of the hypothalamus and brain stem, as shown by magnetic resonance imaging in rats [51]. 
Pharmacological doses of GLP-1 enhance GLP-1 levels in 8 to 10 times, and treatment with these agents decrease gastric emptying in a dose-dependent way, leading to a transient sensation of nausea, vomiting and thus promoting weight loss [52].

GLP-1 receptors are expressed in several regions of the brain, predominantly in hypothalamus and brain stem, where the core regulation of food intake and satiety resides. GLP-1 crosses the blood bran barrier and inhibits food intake by promoting satiety. Animal studies have demonstrated that agonists of the GLP-1 receptor (for instance, liraglutide) were also able to cross the blood brain barrier following peripheral administration, and peripheral administration of GLP-1 suppressed food intake over the dependent and independent pathways of the vagus nerve, resulting in direct action in the brain GLP-1 receptor in the central nervous system [53].

In the same study, liraglutide, increased the CART neurotransmitter expression of the POM/CART anorexigenic neurons in the hypothalamus, increasing energy expenditure and preventing the increase of the expression of orexigenic neurons (NPYIARGP), promoting hunger control, thus avoiding weight gain [53].

One other investigation found out that liraglutide increases energy expenditure, through the activation of the brown adipose tissue (thermogenic tissue) through the central action mechanism in the hypothalamic region, specifically ventromedial nucleus [54].

GLP-1 RAs have also been shown to exert a neuroprotective role in rodents with Alzheimer's and Parkinson's disease [50]. Preclinical studies demonstrated that exendin-4 decreased glutamate beta-amyloid peptide, preventing apoptosis in rat hippocampal neurons in culture. Both glutamate and beta-amyloid peptide is involved in neurodegeneration process [52].

Other studies using GLP-1 RAs involved the autonomic nervous system to mediate the reduction of blood pressure, demonstrating that GLP-1 activation enhances the secretion of atrial natriuretic peptide, relevant to vasodilatation and lowering blood pressure. The GLP-1 physiologic actions in their receptors, including the heart, are not fully understood, but preclinical studies suggest that GLP-1 RAs interact with the heart and blood vessels regardless of their glycemic effect, and that its cardioprotection may occur through a variety of direct and indirect mechanisms.

Preclinical studies using natural GLP-1 and GLP-1 RAs provided good evidences for cardioprotective effects, while clinical trials have shown beneficial effects in hypertension and dyslipidemia in patients with type 2 diabetes [50].

Liraglutide and exenatide have been shown to promote cardioprotective effect in preclinical studies. Liraglutide reduced infarct size and improved survival after myocardial infarction in rats. Similarly, mice fed with high-fat diet had improved cardiac function in treatment with liraglutide [50].

Exenatide also reduced secretion of TAG and ApoB, when administered 1 hour after oral fat load, suggesting that the effect on postprandial lipid metabolism was not related to delayed gastric emptying. Furthermore, exenatide $(0.1 \mathrm{nM})$ decreased ApoB-48 directly in a culture of hamster enterocytes, as assessed by reduced levels of ApoB-48 35S-labeled over a time course of 90 minutes. These findings suggest a key role of GLP-1 in control of chylomicrons secretion, independent of variations in gastric emptying [50].

If the classic GLP-1R is expressed in adipocytes is uncertain. GLP-1 binding sites were detected in the solubilized membranes from both human adipose tissue and rat using radioligand binding assays, although other trials have been unable to detect GLP-1 mRNA expression in adipose tissue of humans and rats [50].

Therefore, the incretin-based therapy may have beneficial effects in various organs, due to the presence of the GLP-1 receptor in many physiologic systems of the human body. These effects are expected to be present in the following systems: cardiovascular system, lipid metabolism, neurological disorders, blood pressure - especially on systolic blood pressure, and in body weight. It is important to point out that there is also the possibility of some non-beneficial effects, such as in the gastrointestinal system and in increased heart rate.

\section{Trials on GLP-1 RAs and Cardiovascular Risk}

Some evidence points to a positive effect of incretin therapy, based on the control of systolic blood pressure. In pooled data from over 2000 patients in 6 exenatide trials, the drug was associated with greater reductions in systolic blood pressure than placebo or insulin for a period of 6 months.

In the clinical study EUREXA, which compared the use of exenatide twice a day with glimepiride in subjects with uncontrolled monotherapy with metformin type 2 diabetes, cardiovascular risk factors, including systolic blood pressure, were significantly improved in subjects using exenatide [50]. 
Although it has a general profile of reducing cardiovascular risk factor, the incretin-based therapy has also been associated with increases in heart rate. In a meta-analysis of 22 clinical studies exenatide and liraglutide were associated with an increase in median heart rate. It is unknown clinical significance of changes in heart rate related to GLP-1 RAs, but it is the current aim in ongoing clinical trials.

Large multicentered studies are currently underway, to confirm the cardiovascular safety use of incretins in sub jects with type 2 diabetes. Among them, LEADER stands out as an important study comparing long term cardiovascular outcomes of liraglutide versus placebo, in patients with type 2 diabetes. This study enrolled 9340 type 2 diabetes patients 50 years of age or older, with a high cardiovascular risk, from August 2010 to November 2015.

EXCEL is a phase 3 study under way, started in 2010 and expected to end in 2018, with an estimate of 14,000 patients enrolled, focused on the analysis of the role of exenatide use once a week in security on major cardiovascular events.

ELIXA, a study conducted with 6000 patients using lixisenatide for shortly over 2 years, published their results on June 2015, showing that the effects of this drug on cardiovascular outcomes in patients with type 2 diabetes after acute coronary syndrome was neutral, which means that there was neither an increase nor a decrease in cardiovascular events among patients using lixisenatide [49]. Additionally, no increase in pancreatitis or pancreas cancer was detected.

Just as it goes with the effects on blood glucose and blood pressure, the potential effects of GLP-1 RAs in triglycerides and other lipids parameters may be related with the simultaneous weight loss. However, the effects on blood glucose, pancreatic hormones and motility might also contribute to improvement in lipid profile, and it is possible that the incretin-based therapy may a direct influence on the intestinal metabolism of lipids, particularly inhibiting the production of triglyceride-rich lipoproteins.

An aggressive intervention in dyslipidemia among patients with type 2 diabetes has an important impact in preventing cardiovascular complications. The reductions in triglycerides and free fatty acids concentrations were observed with the first infusion of natural GLP-1 [49]. Exenatide, administered twice a day, and liraglutide, once a day, were associated with $20 \%$ or more reductions in triglyceride levels [52].

A meta-analysis of LEADER trials found that the treatment with liraglutide significantly reduced total cholesterol, LDL cholesterol, free fatty acids and triglycerides as compared to baseline ( $P<0.01$, to all changes). It is important to point out that LEADER enrolled high-risk patients with type 2 diabetes and a mean age of 64.3 years of age [49].

\section{Weight Loss Associated with the Use of GLP-1 Receptor Agonists}

The World Health Organization estimates that 1.5 billion adults worldwide are overweight and 500 million are obese. Almost three million adults die each year as a result of being overweight or obese [55]. Overweight and obesity lead to numerous adverse conditions including type 2 diabetes, cardiovascular disease, stroke, and certain cancers [56]. The risk of developing diabetes escalates with the degree of excess body weight, increasing threefold with a body mass index of 25.0 to 29.9, and 20-fold with an index of 35 and higher compared with a healthy index of 18.5 - 24.9 [1]. Weight loss of 5\% to 10\% can improve type 2 diabetes and related comorbidities [57].

The worldwide spread of obesity and associated comorbidities not only threatens quality of life but also presents a significant economic burden. It has been projected that by 2030, costs associated with overweight and obesity will comprise $16 \%$ - 18\% of total health care expenses in the US [56]. Obesity treatment guidelines recommend the use of pharmacologic therapy in adults who have a body mass index (BMI) of $30 \mathrm{~kg} / \mathrm{m}^{2}$ or higher or in patients with a BMI of $27 \mathrm{~kg} / \mathrm{m}^{2}$ or higher who have at least one weight-related comorbid condition (e.g., hypertension, dyslipidemia, insulin resistance, type 2 diabetes mellitus) [58]. While bariatric surgery has proven to be a viable treatment option for the morbidly obese, there is clearly a need for less invasive alternatives [56]. The development of effective pharmacological treatments for obesity has thus far proven unsuccessful [59]. Few safe, effective weight-management drugs are currently available [57]. Recent research has suggested that longacting analogs of the gut hormone, glucagon-like peptide 1 (GLP-1), may have potential as an antiobesity treatment [56].

GLP-1 is an incretin hormone synthesized and secreted principally from two locations: the L cells in the small intestine and neurons in the nucleus tractus solitarius (NTS) of the hindbrain. In addition to glycemic control, the 
GLP-1 system is also involved in regulating food intake in both human and animal models. However, clinical use of the native peptide for obesity and hyperglycemia is limited by its rapid degradation rate by the dipeptidyl-peptidase-4 (DPP-IV) enzyme and other endopeptidases. Longer-acting pharmacological GLP-1 RAs that are resistant to enzymatic degradation, including exendin-4 (trade name Byetta) and liraglutide (trade name Victoza) are currently approved for human type 2 Diabetes Mellitus (T2DM) treatment and may also hold promise for obesity treatment [59]. Several other GLP-1 analogs are currently under investigation or are approved for the treatment of type 2 diabetes. These include lixsenatide (Sanofi Aventis, trade name Lyxumia) and albiglutide (GlaxoSmithKline, trade names Epezan and Tanseum) which have amino acid modifications to resist degradation by DPP-IV. The recently FDA-approved drug, dulaglutide (Eli Lilly, trade name Trulicity), an analog of human GLP-1, is covalently linked to an Fc fragment of human IgG4 enabling it to resist degradation and reduce renal clearance. These analogs were initially used as drugs for the treatment of type 2 diabetes; however, results from clinical trials have repeatedly demonstrated their ability to induce weight loss [57].

The glucagon-like-peptide-1 receptor (GLP-1R) agonists, liraglutide (Victoza) and the synthetic product of exendin-4 (Byetta), are approved for type II diabetes mellitus (T2DM) treatment and may be efficacious in obesity treatment as well, in part, due to the drugs' resistance to enzymatic degradation and prolonged half-life relative to endogenous GLP-1. In the study "Comparative effects of the long-acting GLP-1 receptor ligands, liraglutide and exendin-4, on food intake and body weight suppression in rats", showed that the administration of the GLP-1R ligands, exendin-4 (b.i.d.) and liraglutide (q.d.), lead to comparable and pronounced suppression of food intake and body weight in DIO rats, suggesting a potential role for these drugs as a clinical tool for obesity treatment [60].

Liraglutide is a glucagon-like peptide-1 receptor agonist that has been successfully used in the treatment of type 2 diabetes for several years. Weight loss has been well described as an additional benefit with liraglutide therapy, which prompted the manufacturer to evaluate and develop a higher dose formulation specifically for the treatment of obesity. Liraglutide $3 \mathrm{mg} /$ day (trade name Saxanda) was approved by the U.S. Food and Drug Administration for this indication in December 2014 [58]. This drug was approved for adults with a body mass index (BMI) of 30 or higher or those with a BMI of 27 or higher who have at least one weight-related comorbid condition such as hypertension, type 2 diabetes, or elevated cholesterol [56]. Data demonstrated that liraglutide can successfully achieve weight-loss benchmarks of $5 \%$ or more and $10 \%$ or more loss from baseline. The most common adverse effects were gastrointestinal and mild to moderate in intensity. Liraglutide $3 \mathrm{mg} / \mathrm{day}$ represents another pharmacologic option for the treatment of obesity [58]. This drug was approved on December 2014 by the US Food and Drug Administration as an obesity treatment option and shown in clinical trials to be effective in reducing and sustaining body weight loss [56].

The study "Efficacy of Liraglutide for Weight Loss Among Patients With Type 2 Diabetes", one randomized clinical trial to investigate efficacy and safety of liraglutide vs placebo for weight management in adults with overweight or obesity and type 2 diabetes, showed that the use of subcutaneous liraglutide $(3.0 \mathrm{mg})$ daily, compared with placebo, resulted in weight loss over 56 weeks. Weight loss was 6.0\% (6.4 kg) with liraglutide (3.0$\mathrm{mg}$ dose), 4.7\% (5.0 kg) with liraglutide (1.8-mg dose), and 2.0\% (2.2 kg) with placebo. Weight loss of 5\% or greater occurred in $54.3 \%$ with liraglutide $(3.0 \mathrm{mg}$ ) and $40.4 \%$ with liraglutide $(1.8 \mathrm{mg}$ ) vs $21.4 \%$ with placebo. Weight loss greater than $10 \%$ occurred in $25.2 \%$ with liraglutide $(3.0 \mathrm{mg})$ and $15.9 \%$ with liraglutide $(1.8 \mathrm{mg})$ vs $6.7 \%$ with placebo. More gastrointestinal disorders were reported with liraglutide (3.0 mg) vs liraglutide (1.8 $\mathrm{mg}$ ) and placebo. No pancreatitis was reported in this study. Among overweight and obese participants with type 2 diabetes, use of subcutaneous liraglutide $(3.0 \mathrm{mg}$ ) daily, compared with placebo, resulted in weight loss over 56 weeks [57].

A recent comparison between the clinical efficacy of bariatric surgery and liraglutide found surgery to be superior to liraglutide for both body weight reduction and improvements in metabolic parameters. Despite this, many individuals are not ideal candidates for surgery and would benefit from noninvasive medical treatments. Liraglutide has been demonstrated to produce significant weight loss in humans, with and without type 2 diabetes, while producing minimal side effects and thus is an attractive treatment option. Additional research is warranted for its potential in combinatorial weight loss treatments that may further enhance and sustain weight loss [56].

A relevant systematic review called "Effects of glucagon-like peptide-1 receptor agonists on weight loss" is a meta-analysis of randomised controlled trials conducted with patients showing a body mass index of 25 or higher, with or without type 2 diabetes mellitus, receiving exenatide twice daily, exenatide once weekly, or liraglu- 
tide once daily at clinically relevant doses for at least 20 weeks. GLP-1R agonist groups achieved a greater weight loss than control groups (mean difference $-2.9 \mathrm{~kg}, 95 \%$ confidence interval -3.6 to $-2.2 ; 21$ trials, 6411 participants). The results showed weight loss in the GLP-1R agonist groups for patients without diabetes ( -3.2 $\mathrm{kg},-4.3$ to -2.1 ; three trials) as well as in patients with diabetes ( $-2.8 \mathrm{~kg},-3.4$ to $-2.3 ; 18$ trials). In patients with type 2 diabetes, improved glycemic control was often associated with increased body weight. The participants without diabetes achieved greater reductions in body weight than those with diabetes. The effects of GLP-1 RAs were more pronounced in patients without diabetes. In the overall analysis, GLP-1 RAs had beneficial effects on systolic and diastolic blood pressure, plasma concentrations of cholesterol, and glycaemic control, and did not have a significant effect on plasma concentrations of liver enzymes. GLP-1 RAs were associated with nausea, diarrhoea, and vomiting, but not with hypoglycaemia. Adverse events became more frequent in higher doses. This review provides evidence that treatment with GLP-1 Ras leads to weight loss in overweight or obese patients, with or without type 2 diabetes mellitus [55].

The FDA-approved GLP-1 ARs agonists exendin-4 and liraglutide reduce food intake and body weight. Nausea is the most common adverse side effect reported with these GLP-1R agonists. Whether food intake suppression by exendin-4 and liraglutide occurs independently of nausea is unknown. Further, the neurophy-siological mechanisms mediating the nausea associated with peripheral GLP-1R agonist use are poorly understood [59]. Delays in gastric emptying may partially contribute to the reduction in meal intake produced by liraglutide in humans [37]. The food intake suppression by the GLP-1 RAs is accompanied by nausea. The nauseating effects accompanying peripheral exendin- 4 are mediated by a vagal-independent pathway that appears to involve BBB penetrance and subsequent GLP-1R activation in the CNS. The GLP-1R expressed within the mNTS and potentially in other, yet undefined GLP-1R brain nuclei are likely key contributors to both the food intake suppressive and nauseating effects of these long-acting GLP-1 RAs [59].

The mechanisms by which long-acting GLP-1 receptor agonists reduce food intake and body weight are actively under investigation. One potential mechanism for reducing body weight is through GLP-1's effects on energy expenditure. Central injection of liraglutide in rodents stimulates brown-fat thermogenesis and induces browning of white adipose tissue independent of its ability to suppress food intake [37]. In rodents, the long-term liraglutide treatment significantly increased expression of cocaine and amphetamine-related transcript but did not increase neuropeptide Y (NPY)/agouti-related protein (AgRP) gene expression in the arcuate nucleus suggesting that liraglutide prevents the normal activation of the NPY/AgRP system to increase food intake during periods of weight loss. Preventing the normal stimulation of food intake by NPY/AgRP that occurs during energy deficit would benefit sustained decreases in body weight [56].

An interaction between the adipose-derived peptide, leptin, and GLP-1 has been demonstrated in experiments in rodents showing that subthreshold doses of leptin increased the ability of GLP-1 inhibit food intake. Furthermore, GLP-1 receptor agonists can reinstate leptin sensitivity in obese animals. As in animals, obese humans have high levels of circulating leptin but are leptin resistant and therefore do not respond appropriately to this signal to reduce energy intake. During weight loss, a drop in plasma leptin levels that results from decreased fat mass produces a rebound response that favors increased food intake. Based on animal studies, it was postulated that administration of a GLP-1 receptor agonist during diet-induced weight loss would inhibit decreases in soluble leptin receptor plasma concentrations (one marker for leptin activity) caused by decreased adiposity and thus prevent a regain in body weight. This study demonstrated that liraglutide $(1.2 \mathrm{mg} / \mathrm{day})$ inhibited the increase in soluble leptin receptor that is normally seen in individuals undergoing weight loss. Consequently, free leptin levels were increased, thereby providing a signal that would prevent an increase in food intake and a decrease in energy expenditure. Supporting the hypothesis, the results indicated that the group receiving the liraglutide, in conjunction with reduced caloric intake, had greater weight loss during the maintenance period than the group that did not receive liraglutide. This study has important implications for the potential use of liraglutide as an adjunct to more traditional weight loss regimens and for the long-term sustainability of weight loss treatments. Additionally, it suggests another avenue through which GLP-1 receptor agonists contribute to weight loss [56].

The GLP-1 RAs have been demonstrated to reduce food intake, promote weight loss, and improve indices of metabolic function in both animal and human studies. The primary mechanisms associated with these effects are proposed to be due to actions of GLP-1 on peripheral (vagal) and central pathways that affect food intake and metabolism via hindbrain and hypothalamic activation, as well as those brain areas associated with motivation and reward processes [56]. 


\section{Side Effects and Associated Risks of GLP-1 Agonists Receptor}

\section{1) Gastrointestinal Effects}

The most common adverse reactions found from the use of GLP-1 RAs are gastrointestinal motility disorders, such as nausea, vomiting and diarrhea, due to the mechanism of action of GLP-1. These side effects often lead to discontinuation of treatment by the patient [61]. They were the most commonly observed effect, and its occurrence varied from $10 \%$ to $42 \%$ of patients, as the different doses were used in trials. Gastrointestinal side effects were more common in the high-dose regimens and tend to improve with use over time [62]. The slow and gradual titration of the dose, over the first few weeks of treatment, often helps reducing the effects [55]. However, despite common, such reactions do not imply concrete health risks to the patient [61].

2) Acute Pancreatitis

GLP-1 RAs are associated with an increased risk of acute pancreatitis in diabetic patients [63]. Pancreatitis cases have been reported in animals and in humans treated with agents of this class. Exenatide and Liraglutide are associated with reported cases of acute pancreatitis, whereas lixisenatide has not, so far [64]. Studies show an average incidence of 1.6 cases of acute pancreatitis per 1000 patients/years of exposure, as for Liraglutide [65].

Many cases of pancreatitis have been reported in patients treated with exenatide, leading to the conduction informative and regulatory reviews [66]. Data from animal exposure studies, as well as several clinical trials in over 28,000 patients that received some type of incretin-based therapy, have been extensively reviewed by the US Food and Drug Administration (FDA) and the European Medicine Agency (EMA). Nonetheless, even after all those data raised to date, pancreatitis remains as a risk associated with the use of such drugs until new studies that clearly demonstrate a safety profile are available [66]. The latest guidelines still recommend the careful use of GLP-1 RAs in patients with a historic of pancreatic disease and suspending it if acute pancreatitis develops during the drug use. Besides, all patients in use of GLP-1 RAs should be advised of the increased risk of pancreatitis associated with liraglutide and exenatide use, which may be a class effect [67].

3) Medular Thyroid Carcinoma

GLP-1 receptors are expressed in thyroid tissue, especially in C cells [63] [68]. In animal studies in preclinical stage, there was an increase in C cell hyperplasia frequency and thyroid cancer among mice and rats exposed to liraglutide [48]. While stimulating the GLP-1 receptor induces the release of calcitonin in rodents, it is unknown if GLP-1 RAs are associated with a raised incidence of medullary thyroid carcinoma MTC) in humans, implying potential species-specific differences in the GLP-1 receptor expression in the thyroid. Rodents have significantly more $\mathrm{C}$ cell receptors than humans, which could justify the increase of thyroid disorders in these animal studies [63] [69].

Medullary thyroid carcinoma cases were reported in patients in use of liraglutide. These cases are not sufficient to establish or to deny a causal association [70] [71]. To date, the FDA and the European Medical Association have not reach a final conclusion regarding this possible association, as expressed on a 2014 panel published at the New England Journal of Medicine. FDA still recommends specific monitoring of MTC in patients on GLP-1 RAs therapy, and these drugs are currently contraindicated in patients with a family history of MEN 2 or MTC [67].

4) Renal Effects

The experience of the use of GLP-1 receptor in patients with chronic kidney disease (CKD) is still very limited. Exenatide has renal elimination and should be avoided in patients with CKD [72]. Liraglutide has no renal excretion, but should be used with caution in patients with CKD, due to the necessity of more studies confirming their safety [73]. The available data are still very limited on lixisenatide, long-acting exenatide and other weekly applicable drugs that are in development [65] [73]. Many case reports of acute renal failure have been reported with the use of GLP-1 agonists, most likely triggered by dehydration from the gastrointestinal adverse effects-interpret vomiting and diarrhea [65] [73]. Thus, until more data are available, it is recommended the cautious use of this drug class in patients with CKD [65].

5) Hypoglycemia

Hypoglycemia is not a prominent effect of treatment with GLP-1 agonists. In general, this only occurs when there is concomitant use of insulin or sulfonylureas [55] or if a reduction of medication doses is not thought of, when starting a new antidiabetic drug [74] [75]. Therefore, it is recommended to lower the dose of these agents in order to add a GLP-1 RA [75]. 
6) Antibodies Formation

GLP-1 RAs are peptide agents. Thus, there is a concern that anti-drug antibodies may appear, leading to decreased efficiency and increased hypersensitivity reactions over time [71].

Antibody levels have been measured in clinical trials, with great variation among class agents, which might represent differences in immunogenicity of the formulations. The newer formulations, including albiglutide and dulaglutide, show a lower risk of antibody formation, when compared to exenatide and liraglutide [76]. Exenatide is a GLP-1 RA that leads to the formation of antibodies, probably due to lower homology to native GLP-1 [76]. Liraglutide is less immunogenic than exenatide [77]. In clinical trials, patients who had the highest titles of anti-drug antibodies had worse performance in reducing A1c hemoglobin [78].

7) Local Reactions

Skin reactions at the injection site were observed in patients with positive anti-drug antibody titration [71]. The long-duration agents exenatide LAR and albiglutide have a significant incidence of such reactions, while liraglutide, lixisenatide and daily application exenatide do not present these problems. Interestingly, dulaglutide, despite the long-term action (weekly application), showed no significant levels of local reactions [61].

\section{Conclusions}

Incretin-based therapy may have beneficial effects on various organs and metabolic mechanisms, due to the presence of the GLP-1 receptor in many physiologic systems of the human body. These effects are expected the following systems: cardiovascular system, lipid metabolism, neurological disorders, blood pressure-especially on systolic blood pressure, and in body weight. It is important to point out that there is also the possibility of some non-beneficial effects, such as in the gastrointestinal system and in increased heart rate.

Treatment with GLP-1 receptor agonists may enhance the endogenous secretion of insulin induced by meal ingestion and inhibit glucagon secretion, thereby improving glucose homoeostasis. Notably, it also suppresses food intake and appetite. Trials of patients with type 2 diabetes suggest that agonists of GLP-1 receptor (GLP1R) have beneficial effects on metabolic regulation and lead to weight loss. Besides its presence in the gastrointestinal tract, GLP-1 is found in the central nervous system localized to neurons primarily in the nucleus of the solitary tract (NTS) in the caudal brainstem. Neurons in the NTS that express GLP-1 send projections to several brain regions that take part in feeding behavior and energy homeostasis.

Thus, GLP-1 receptor antagonists show broad metabolic effects, far beyond the glycemic control, and represent effective therapies for type 2 diabetes. Further benefits on metabolism are being discovered, as new trials come out. The next few years are expected to bring relevant new data regarding extra glycemic effects of GLP-1 receptor agonists.

\section{Acknowledgements}

We thank Prof. Aline Marcadenti for her unique dedication to research.

We would also like to thank IPEMED Brazil for supporting continuous research towards a better medical education in Brazil and abroad.

\section{References}

[1] Deacon, C.F., Hughes, T.E. and Holst, J.J. (1998) Dipeptidyl Peptidase IV Inhibition Potentiates the Insulinotropic Effect of Glucagon-Like Peptide 1 in the Anesthetized pig. Diabetes, 47, 764-769. http://dx.doi.org/10.2337/diabetes.47.5.764

[2] Wei, Y. and Mojsov, S. (1995) Tissue-Specific Expression of the Human Receptor for Glucagon-Like Peptide I: Brain, Heart and Pancreatic Forms Have the Same Deduced Amino Acid Sequences. FEBS Letters, 358, 219-224. http://dx.doi.org/10.1016/0014-5793(94)01430-9

[3] Fehmann, H.C. and Habener, J.F. (1992) Insulinotropic Hormone Glucagon-Like Peptide-I (7-37) Stimulation of Proinsulin Gene Expression and Proinsulin Biosyn-Thesis in Insulinoma Beta TC-1 Cells. Endocrinology, 130, 159-166.

[4] Buteau, J., Roduit, R., Susini, S. and Prentki, M. (1999) Glucagon-Like Peptide-1 Promotes DNA Synthesis, Activates Phosphatidylinositol 3-Kinase and Increases Transcription Factor Pancreatic and Duodenal Homeobox Gene 1 (PDX-1) DNA Binding Activity in Beta (INS-1)-Cells. Diabetologia, 42, 856-864. http://dx.doi.org/10.1007/s001250051238

[5] Stoffers, D.A., Kieffer, T.J., Hussain, M.A., Drucker, D.J., Bonner-Weir, S., Habener, J.F. and Egan, J.M. (2000) Insulinotropic Glucagon-Like Peptide 1 Agonists Stimulate Expression of Homeodomain Protein IDX-1 and Increase Islet 
Size in Mousepancreas. Diabetes, 49, 741-748. http://dx.doi.org/10.2337/diabetes.49.5.741

[6] Nauck, M.A. (2009) Unraveling the Science of Incretin Biology. The American Journal of Medicine, 122, S3-S10. http://dx.doi.org/10.1016/j.amjmed.2009.03.012

[7] Drucker, D.J. and Nauck, M.A. (2006) The Incretin System: Glucagon-Like Peptide-1 Receptor Agonists and Dipeptidyl Peptidase-4 Inhibitors in Type 2 Diabetes. The Lancet, 368, 1696-1705. http://dx.doi.org/10.1016/S0140-6736(06)69705-5

[8] Kendall, D.M., Cuddihy, R.M. and Bergenstal, R.M. (2009) Clinical Application of Incretin-Based Therapy: Therapeutic Potential, Patient Selection and Clinical Use. The American Journal of Medicine, 122, S37-S50. http://dx.doi.org/10.1016/j.amjmed.2009.03.015

[9] Bidcnsdottir, I., Olsen, A., Larsen, U., et al. (2008) Metabolism and Excretion of the Once-Daily Human GLP-l Analogue Liraglutide in Healthy Subjects and Its in Vitro Degradation by Dipeptidyl Peptidase IV and Neutral Endopeptidase. Diabetologia, 51, S356.

[10] Agerso, H., Jensen, L.B., Elbrend, B., Rolan, P. and Zdravkovic, M. (2002) The Pharmacokinetics, Pharmacodynaruics, Safety and Tolerability of NN2211, a New Long-Acting GLP-l Derivative, in Healthy men. Diabetologia, 45, $195-202$. http://dx.doi.org/10.1007/s00125-001-0719-z

[11] Cilia, C.W. and Egan, J.M. (2008) Incretin-Based Therapies in Type 2 Diabetes Mellitus. The Journal of Clinical Endocrinology \& Metabolism, 93, 3703-3716. http://dx.doi.org/10.1210/jc.2007-2109

[12] Kolterman, O.G., Buse, J.B., Fineman, M.S., Gaines, E., Heintz, S., Bicsak, T.A., Taylor, K., Kim, D., Aisporna, M., Wang, Y. and Baron, A.D. (2003) Synthetic Exendin-4 (Exenatide) Significantly Reduces Postprandial and Fasting Plasma Glucose in Subjects with Type 2 Diabetes. The Journal of Clinical Endocrinology \& Metabolism, 88, 30823089. http://dx.doi.org/10.1210/jc.2002-021545

[13] Zander, M., Madsbad, S., Madsen, J.L. and Holst, J.J. (2002) Effect of 6-Week Course of Glucagon-Like Peptide 1 on Glycaemic Control, Insulin Sensitivity, and $\beta$-Cell Function in Type 2 Diabetes: A Parallel-Group Study. The Lancet, 359, 824-830. http://dx.doi.org/10.1016/S0140-6736(02)07952-7

[14] Göke, R., Fehmann, H.C., Linn, T., Schmidt, H., Krause, M., Eng, J. and Göke, B. (1993) Exendin-4 Is a High Potency Agonist and Truncated Exendin-(9-39)-Amide an Antagonist at the Glucagon-Like Peptide 1-(7-36)-Amide Receptor of Insulin-Secreting $\beta$-Cells. The Journal of Biological Chemistry, 268, 19650-19655.

[15] Nielsen, L.L., Young, A.A. and Parkes, D. (2004) Pharmacology of Exenatide (Synthetic Exendin-4): A Potential Therapeutic for Improved Glycemic Control of Type 2 Diabetes. Regulatory Peptides, 117, 77-88. http://dx.doi.org/10.1016/j.regpep.2003.10.028

[16] Nielsen, L.L. and Baron, A.D. (2003) Pharmacology of Exenatide (Synthetic Exendin-4) for the Treatment of Type 2 Diabetes. Current Opinion In Investigational Drugs, 4, 401-405.

[17] Young, A.A., Gedulin, B.R., Bhavsar, S., Bodkin, N., Jodka, C., Hansen, B. and Denaro, M. (1999) Glucose-Lowering and Insulin-Sensitizing Actions of Exendin-4: Studies in Obese Diabetic (ob/ob, db/db) Mice, Diabetic Fatty Zucker Rats, and Diabetic Rhesus Monkeys (Macaca mulatta). Diabetes, 48, 1026-1034. http://dx.doi.org/10.2337/diabetes.48.5.1026

[18] Szayna, M., Doyle, M.E., Betkey, J.A., Holloway, H.W., Spencer, R.G.S., Greig, N.H. and Egan, J.M. (2000) Exendin-4 Decelerates Food Intake, Weight Gain, and Fat Deposition in Zucker Rats. Endocrinology, 141, 1936-1941. http://dx.doi.org/10.1210/endo.141.6.7490

[19] Gedulin, B.R., Nikoulina, S.E., Smith, P.A., Gedulin, G., Nielsen, L.L., Baron, A.D., Parkes, D. and Young, A.A. (2005) Exenatide (Exendin-4) Improves Insulin Sensitivity and $\beta$-Cell Mass in Insulin-Resistant Obese fa/fa Zucker Rats Independent of Glycemia and Body Weight. Endocrinology, 146, 2069-2076. http://dx.doi.org/10.1210/en.2004-1349

[20] Wang, M., Kipnes, M.S., Matheson, S. and Wen, S. (2007) Safety and Pharmacodynamics of CJC-1134-PC, a Novel GLP-1 Receptor Agonist, in Patients with Type 2 Diabetes Mellitus: A Randomized, Placebo-Controlled, DoubleBlind, Dose-Escalation Study. American Diabetes Association, Chicago.

[21] Drucker, D.J., Buse, J.B., Taylor, K., Kendall, D.M., Trautmann, M., Zhuang, D. and Porter, L., DURATION-1 Study Group (2008) Exenatide Once Weekly versus Twice Daily for the Treatment of Type 2 Diabetes: A Randomised, Open-Label, Non-Inferiority Study. The Lancet, 372, 1240-1250. http://dx.doi.org/10.1016/S0140-6736(08)61206-4

[22] Buse, J.B., Drucker, D.J., Taylor, K.L., Kim, T., Walsh, B., Hu, H., Wilhelm, K., Trautmann, M., Shen, L.Z. and Porter, L.E., DURATION-1 Study Group (2010) DURATION-1: Exenatide Once Weekly Produces Sustained Glycemic Control and Weight Loss over 52 Weeks. Diabetes Care, 33, 1255-1261. http://dx.doi.org/10.2337/dc09-1914

[23] Bergenstal, R.M., Wysham, C., Macconell, L., Malloy, J., Walsh, B., Yan, P., Wilhelm, K., Malone, J. and Porter, L.E., DURATION-2 Study Group (2010) Efficacy and Safety of Exenatide Once Weekly versus Sitagliptin or Pioglitazone as an Adjunct to Metformin for Treatment of Type 2 Diabetes (DURATION-2): A Randomised Trial. Lancet, 376, 
431-439. http://dx.doi.org/10.1016/S0140-6736(10)60590-9

[24] Seewoodhary, J. (2011) Novel GLP-1 Mimetics in Diabetes: Lixisenatide and Albiglutide. Future Prescriber, 12, 20-22. http://dx.doi.org/10.1002/fps.79

[25] Barrington, P., Chien, J.Y., Showalter, H.D., Schneck, K., Cui, S., Tibaldi, F., et al. (2011) A 5-Week Study of the Pharmacokinetics and Pharmacodynamics of LY2189265, a Novel, Long-Acting Glucagon-Like Peptide-1 Analogue, in Patients with Type 2 Diabetes. Diabetes, Obesity and Metabolism, 13, 426-433. http://dx.doi.org/10.1111/j.1463-1326.2011.01364.x

[26] Thorkildsen, C., Neve, S., Larsen, B.D., Meier, E. and Petersen, J.S. (2003) Glucagon-Like Peptide 1 Receptor Agonist ZP10A Increases Insulin mRNA Expression and Prevents Diabetic Progression in $\mathrm{db} / \mathrm{db}$ Mice. Journal of Pharmacology and Experimental Therapeutics, 307, 490-496. http://dx.doi.org/10.1124/jpet.103.051987

[27] Haschke, G., Haag-Diergarten, S., Werner, U., Kramer, W. and Herling, A.W. (2006) The GLP-1 Receptor Agonist AVE0010 Preserves Beta Cell Function and Insulin Secretion after a Week Treatment in Male Obese Zucker Diabetic Fatty Rats-An Isolated Perfused Pancreas Study [Abstract]. Diabetologia, 49, 400-401.

[28] Werner, U., Haschke, G., Herling, A.W. and Kramer, W. (2010) Pharmacological Profile of Lixisenatide: A New GLP-1 Receptor Agonist for the Treatment of Type 2 Diabetes. Regulatory Peptides, 164, 58-64. http://dx.doi.org/10.1016/j.regpep.2010.05.008

[29] Bush, M.A., Matthews, J.E., De Boever, E.H., Dobbins, R.L., Hodge, R.J., Walker, S.E., Holland, M.C., Gutierrez, M. and Stewart, M.W. (2009) Safety, Tolerability, Pharmacodynamics and Pharmacokinetics of Albiglutide, a Long-Acting Glucagon-Like Peptide-1 Mimetic, in Healthy Subjects. Diabetes, Obesity and Metabolism, 11, 498-505. http://dx.doi.org/10.1111/j.1463-1326.2008.00992.x

[30] Baggio, L.L., Huang, Q., Brown, T.J. and Drucker, D.J. (2004) A Recombinant Human Glucagon-Like Peptide (GLP)1-Albumin Protein (Albugon) Mimics Peptidergic Activation of GLP-1 Receptor-Dependent Pathways Coupled with Satiety, Gastrointestinal Motility, and Glucose Homeostasis. Diabetes, 53, 2492-2500. http://dx.doi.org/10.2337/diabetes.53.9.2492

[31] Bloom, M., Bock, J., Duttaroy, A., Grzegorzewski, K., Moore, P., Ou, Y., Wojcik, S., Zhou, X. and Bell, A.C. (2003) Albugon Fusion Protein: A Long-Acting Analog of GLP-1 That Provides Lasting Antidiabetic Effect in Animals (Abstract). Diabetes, 52, A112.

[32] Ou, Y.C., Bloom, M., Grzegorzewski, K.J., Bock, J., Duttaroy, A., Moore, P., Wojcik, S., Zhou, J.X., Sung, C. and Bell, A.C. (2003) Pharmacokinetic and Pharmacodynamic Analysis of Albugon, a Long-Acting Analog of Glucagon-Like Peptide-1, in Mice and Monkeys (Abstract). AAPS PharmSci, 5, 5263.

[33] Rosenstock, J., Reusch, J., Bush, M., Yang, F. and Stewart, M. (2009) Potential of Albiglutide, a Long-Acting GLP-1 Receptor Agonist, in Type 2 Diabetes: A Randomized Controlled Trial Exploring Weekly, Biweekly, and Monthly Dosing. Diabetes Care, 32, 1880-1886. http://dx.doi.org/10.2337/dc09-0366

[34] Dungan, K.M., Povedano, S.T., Forst, T., González González, J.G., Atisso, C., Sealls, W. and Fahrbach, J.L. (2014) Once-Weekly Dulaglutide versus Once-Daily Liraglutide in Metformin-Treated Patients with Type 2 Diabetes (AWARD-6): A Randomised, Open-Label, Phase 3, Non-Inferiority Trial. The Lancet, 384, 1349-1357. http://dx.doi.org/10.1016/S0140-6736(14)60976-4

[35] Lund, A., Knop, F.K. and Vilsboll, T. (2014) Glucagon-Like Peptide-1 Receptor Agonists for the Treatment of Type 2 Diabetes: Differences and Similarities. European Journal of Internal Medicine, 25, 407-414. http://dx.doi.org/10.1016/j.ejim.2014.03.005

[36] Kendall, D.M., Riddle, M.C., Rosenstock, J., Zhuang, D., Kim, D.D., Fineman, M.S. and Baron, A.D. (2005) Effects of Exenatide (Exendin-4) on Glycemic Control over 30 Weeks in Patients with Type 2 Diabetes Treated with Metformin and a Sulfonylurea. Diabetes Care, 28, 1083-1091. http://dx.doi.org/10.2337/diacare.28.5.1083

[37] Garber, A.J., Abrahamson, M.J., Barzilay, J.I., Blonde, L., Bloomgarden, Z., Bush, M., et al. (2013) American Association of Clinical Endocrinologists' Comprehensive Diabetes Management Algorithm 2013 Consensus Statement-Executive Summary. Endocrine Practice, 19, 536-557. http://dx.doi.org/10.4158/EP13176.CS

[38] Inzucchi, S.E., Bergenstal, R.M., Buse, J.B., Diamant, M., Ferrannini, E., Nauck, M., et al. (2012) Management of Hyperglycaemia in Type 2 Diabetes: A Patient-Centered Approach. Position Statement of the American Diabetes Association (ADA) and the European Association for the Study of Diabetes (EASD). Diabetologia, 55, 1577-1596. http://dx.doi.org/10.1007/s00125-012-2534-0

[39] Zoungas, S., Chalmers, J., Ninomiya, T., Li, Q., Cooper, M.E., Colagiuri, S., et al., ADVANCE Collaborative Group (2012) Association of HbA1c Levels with Vascular Complications and Death in Patients with Type 2 Diabetes: Evidence of Glycaemic Thresholds. Diabetologia, 55, 636-643. http://dx.doi.org/10.1007/s00125-011-2404-1

[40] Buse, J.B., Rosenstock, J., Sesti, G., Schmidt, W.E., Montanya, E., Brett, J.H., Zychma, M. and Blonde, L., LEAD-6 Study Group (2009) Liraglutide Once a Day versus Exenatide Twice a Day for Type 2 Diabetes: A 26-Week Rando- 
mised, Parallel-Group, Multinational, Open-Label Trial (LEAD-6). Lancet, 374, 39-47. http://dx.doi.org/10.1016/S0140-6736(09)60659-0

[41] Garber, A., Henry, R.R., Ratner, R., Hale, P., Chang, C.T. and Bode, B. (2011) Liraglutide, a Once-Daily Human Glucagon-Like Peptide 1 Analogue, Provides Sustained Improvements in Glycaemic Control and Weight for 2 Years as Monotherapy Compared with Glimepiride in Patients with Type 2 Diabetes. Diabetes, Obesity and Metabolism, 13, 348-356. http://dx.doi.org/10.1111/j.1463-1326.2010.01356.x

[42] Meier, J.J. (2012) GLP-1 Receptor Agonists for Individualized Treatment of Type 2 Diabetes Mellitus. Nature Reviews Endocrinology, 8, 728-742. http://dx.doi.org/10.1038/nrendo.2012.140

[43] Buse, J.B., Nauck, M., Forst, T., Sheu, W.H.-H., Shenouda, S.K., Heilmann, C.R., et al. (2013) Exenatide Once Weekly versus Liraglutide Once Daily in Patients with Type 2 Diabetes (DURATION-6): A Randomised, Open-Label Study. Lancet, 381, 117-124. http://dx.doi.org/10.1016/S0140-6736(12)61267-7

[44] Rosenstock, J., Raccah, D., Koranyi, L., Maffei, L., Boka, G., Miossec, P. and Gerich, J.E. (2013) Efficacy and Safety of Lixisenatide Once Daily versus Exenatide Twice Daily in Type 2 Diabetes Inadequately Controlled on Metformin: A 24-Week, Randomized, Open-Label, Active-Controlled Study (GetGoal-X). Diabetes Care, 36, 2945-2951. http://dx.doi.org/10.2337/dc12-2709

[45] Pratley, R.E., Nauck, M.A., Barnett, A.H., Feinglos, M.N., Ovalle, F., Harman-Boehm, I., et al., HARMONY 7 Study Group (2014) Once-Weekly Albiglutide versus Once-Daily Liraglutide in Patients with Type 2 Diabetes Inadequately Controlled on Oral Drugs (HARMONY 7): A Randomised, Open-Label, Multicentre, Non-Inferiority Phase 3 Study. The Lancet Diabetes \& Endocrinology, 2, 289-297. http://dx.doi.org/10.1016/S2213-8587(13)70214-6

[46] Wysham, C., Blevins, T., Arakaki, R., Colon, G., Garcia, P., Atisso, C., Kuhstoss, D. and Lakshmanan, M. (2014) Efficacy and Safety of Dulaglutide Added onto Pioglitazone and Metformin versus Exenatide in Type 2 Diabetes in a Randomized Controlled Trial (AWARD-1). Diabetes Care, 379, 2159-2167. http://dx.doi.org/10.2337/dc13-2760

[47] Taylor, K., Gurney, K., Han, J., Pencek, R., Walsh, B. and Trautmann, M. (2011) Exenatide Once Weekly Treatment Maintained Improvements in Glycemic Control and Weight Loss over 2 Years. BMC Endocrine Disorders, 11, 9. http://dx.doi.org/10.1186/1472-6823-11-9

[48] Bunck, M.C., Corner, A., Eliasson, B., Heine, R.J., Shaginian, R.M., Taskinen, M.-R., et al. (2011) Effects of Exenatide on Measures of Beta-Cell Function after 3 Years in Metformin-Treated Patients with Type 2 Diabetes. Diabetes Care, 34, 2041-2047. http://dx.doi.org/10.2337/dc11-0291

[49] Ussher, J. and Drucker, D. (2012) Cardiovascular Biology of the Incretin System. Endocrine Reviews, 33, $187-215$. http://dx.doi.org/10.1210/er.2011-1052

[50] Seufert, J. and Gallwitz, B. (2014) The Extra Pancreatic Effects of GLP-1 Receptor Agonists: A Focus on the Cardiovascular, Gastrointestinal and Central Nervous Systems. Diabetes, Obesity and Metabolism, 16, 673-688. http://dx.doi.org/10.1111/dom.12251

[51] Herzlinger, S. and Horton, E.S. (2013) Extraglycemic Effects of GLP-1 Based Therapautics: Adressing Metabolic and Cardiovascular Risks Associated with Type 2 Diabetes. Diabetes Research and Clinical Practice, 100, 1-10. http://www.sciencedirect.com/science/article/pii/S0168822712004536

[52] Gallwitz, B. (2014) Extra-Pancratic Effects of Incretin-Based Therapies. Endocrine, 47, 360-371.

[53] Baggio, L.L. and Drucker, D.J. (2014) Glucagon Like Peptideo 1 Receptors: Controlling Food Intake and Body Weight. The Journal of Clinical Investigation, 124, 4223-4226. http://dx.doi.org/10.1172/JCI78371

[54] Beiroa, D., Imbernon, M., Gallego, R., Senra, A., Herranz, D., Villarroya, F., et al. (2014) GLP-1 Agonism Stimulates Brown Adipose Tissue Thermogenisisand Browning through Hypothalamic AMPK. Diabetes, 63, 3346-3358. http://dx.doi.org/10.2337/db14-0302

[55] Vilsbøll, T., Christensen, M., Junker, A.E., Knop, F.K. and Gluud, L.L. (2012) Effects of Glucagon-Like Peptide-1 Receptor Agonists on Weight Loss: Systematic Review and Meta-Analyses of Randomised Controlled Trials. BMJ, 344, d7771. http://dx.doi.org/10.1136/bmj.d7771

[56] Ladenheim, E.E. (2015) Liraglutide and Obesity: A Review of the Data So Far. Drug Design, Development and Therapy, 9, 1867-1875. http://dx.doi.org/10.2147/DDDT.S58459

[57] Davies, M.J., Bergenstal, R., Bode, B., Kushner, R.F., Lewin, A., Skjøth, T.V., Andreasen, A.H., Jensen, C.B. and De Fronzo, R.A. (2015) Efficacy of Liraglutide for Weight Loss among Patients with Type 2 Diabetes: The SCALE Diabetes Randomized Clinical Trial. JAMA, 314, 687-699. http://dx.doi.org/10.1001/jama.2015.9676

[58] Nuffer, W.A. and Trujillo, J.M. (2015) Liraglutide: A New Option for the Treatment of Obesity. Pharmacotherapy, 35, 926-934. http://dx.doi.org/10.1002/phar.1639

[59] Kanoski, S.E., Rupprecht, L.E., Fortin, S.M., Jonghe, B.C. and Hayes, M.R. (2012) The Role of Nausea in Food Intake and Body Weight Suppression by Peripheral GLP-1 Receptor Agonists, Exendin-4 and Liraglutide. Neuropharmacol- 
ogy, 62, 1916-1927. http://dx.doi.org/10.1016/j.neuropharm.2011.12.022

[60] Hayes, M.R., Kanoski, S.E., Alhadeff, A.L. and Grill, H.J. (2011) Comparative Effects of the Long-Acting GLP-1 Receptor Ligands, Liraglutide and Exendin-4, on Food Intake and Body Weight Suppression in Rats. Obesity, 19, 1342-1349. http://dx.doi.org/10.1038/oby.2011.50

[61] Tella, S.H. and Rendell, M.S. (2015) Glucagon-Like Polypeptide Agonists in Type 2 Diabetes Mellitus: Efficacy and Tolerability, a Balance. Therapeutic Advances in Endocrinology and Metabolism, 6, 109-134. http://dx.doi.org/10.1177/2042018815580257

[62] Sun, F., Chai, S., Yu, K., Quan, X., Yang, Z., Wu, S., Zhang, Y., Ji, L., Wang, J. and Shi, L. (2015) Gastrointestinal Adverse Events of Glucagon-Like Peptide-1 Receptor Agonists in Patients with Type 2 Diabetes: A Systematic Review and Network Meta-Analysis. Diabetes Technology \& Therapeutics, 17, 35-42. http://dx.doi.org/10.1089/dia.2014.0188

[63] Elashoff, M., Matveyenko, A.V., Gier, B., Elashoff, R. and Butler, P.C. (2011) Pancreatitis, Pancreatic, and Thyroid Cancer with Glucagon-Like Peptide-1-Based Therapies. Gastroenterology, 141, 150-156. http://dx.doi.org/10.1053/j.gastro.2011.02.018

[64] Pappachan, J.M. (2015) Incretin-Based Therapies and Pancreatitis Risk: Myth or Reality. Endocrine, 48, 360-362. http://dx.doi.org/10.1007/s12020-014-0490-9

[65] Scheen, A.J. (2015) Pharmacokinetics and Clinical Use of Incretin-Based Therapies in Patients with Chronic Kidney Disease and Type 2 Diabetes. Clinical Pharmacokinetics, 54, 1-21. http://dx.doi.org/10.1007/s40262-014-0198-2

[66] Egan, A.G., Blind, E., Dunder, K., de Graeff, P.A., Hummer, B.T., Bourcier, T. and Rosebraugh, C. (2014) Pancreatic Safety of Incretin-Based Drugs_FDA and EMA Assessment. New England Journal of Medicine, 370, 794-797. http://dx.doi.org/10.1056/NEJMp1314078

[67] AACE 2015 Guidelines Type 2 Diabetes. https://www.aace.com/files/dm-guidelines-ccp.pdf

[68] Gier, B., Butler, P.C., Lai, C.K., Kirakossian, D., DeNicola, M.M. and Yeh, M.W. (2011) Glucagon Like Peptide-1 Receptor Expression in the Human Thyroid Gland. The Journal of Clinical Endocrinology \& Metabolism, 97, 121-131. http://dx.doi.org/10.1210/jc.2011-2407

[69] Bjerre Knudsen, L., Madsen, L.W., Andersen, S., Almholt, K., de Boer, A.S., Drucker, D.J., et al. (2010) Glucagon-Like Peptide-1 Receptor Agonists Activate Rodent Thyroid C-Cells Causing Calcitonin Release and C-Cell Proliferation. Endocrinology, 151, 1473-1486. http://dx.doi.org/10.1210/en.2009-1272

[70] Hegedüs, L., Moses, A.C., Zdravkovic, M., Le Thi, T. and Daniels, G.H. (2011) GLP-1 and Calcitonin Concentration in Humans: Lack of Evidence of Calcitonin Release from Sequential Screening in over 5000 Subjects with Type 2 Diabetes or Nondiabetic Obese Subjects Treated with the Human GLP-1 Analog, Liraglutide. The Journal of Clinical Endocrinology \& Metabolism, 96, 853-860. http://dx.doi.org/10.1210/jc.2010-2318

[71] Cefalu, W.T., Buse, J.B., Del Prato, S., Home, P.D., LeRoith, D., Nauck, M.A., Raz, I., Rosenstock, J. and Riddle, M.C. (2014) Beyond Metformin: Safety Considerations in the Decision-Making Process for Selecting a Second Medication for Type 2 Diabetes Management Reflections from a Diabetes Care Editors' Expert Forum. Diabetes Care, 37, 2647-2659. http://dx.doi.org/10.2337/dc14-1395

[72] Brunton, S. (2014) GLP-1 Receptor Agonists vs. DPP-4 Inhibitors for Type 2 Diabetes: Is One Approach More Successful or Preferable than the Other? International Journal of Clinical Practice, 68, 557-567. http://dx.doi.org/10.1111/ijcp.12361

[73] Bjerre Knudsen, L., Madsen, L.W., Andersen, S., Almholt, K., de Boer, A.S., Drucker, D.J., et al. (2010) Glucagon-Like Peptide-1 Receptor Agonists Activate Rodent Thyroid C-Cells Causing Calcitonin Release and C-Cell Proliferation. Endocrinology, 151, 1473-1486. http://dx.doi.org/10.1210/en.2009-1272

[74] Zinman, B., Gerich, J., Buse, J.B., Lewin, A., Schwartz, S., Raskin, P., Hale, P.M., Zdravkovic, M., Blonde, L. and The LEAD-4 Study Investigators (2009) Efficacy and Safety of the Human Glucagon-Like Peptide-1 Analog Liraglutide in Combination with Metformin and Thiazolidinedione in Patients with Type 2 Diabetes (LEAD-4 Met+TZD). Diabetes Care, 32, 1224-1230. http://dx.doi.org/10.2337/dc08-2124

[75] Zinman, B., Hoogwerf, B.J., García, S.D., Milton, D.R., Giaconia, J.M., Kim, D.D., Trautmann, M.E. and Brodows, R.G. (2007) The Effect of Adding Exenatide to a Thiazolidinedione in Suboptimally Controlled Type 2 Diabetes: A Randomized Trial. Annals of Internal Medicine, 146, 477-485. http://dx.doi.org/10.7326/0003-4819-146-7-200704030-00003

[76] Garber, A.J. (2011) Long-Acting Glucagon-Like Peptide 1 Receptor Agonists: A Review of Their Efficacy and Tolerability. Diabetes Care, 34, S279-S284. http://dx.doi.org/10.2337/dc11-s231

[77] Buse, J.B., Garber, A., Rosenstock, J., Schmidt, W.E., Brett, J.H., Videbæk, N., Holst, J. and Nauck, M. (2011) Liraglutide Treatment Is Associated with a Low Frequency and Magnitude of Antibody Formation with No Apparent Impact on Glycemic Response or Increased Frequency of Adverse Events: Results from the Liraglutide Effect and Action in 
Diabetes (LEAD) Trials. The Journal of Clinical Endocrinology \& Metabolism, 96, 1695-1702. http://dx.doi.org/10.1210/jc.2010-2822

[78] Fineman, M.S., Mace, K.F., Diamant, M., Darsow, T., Cirincione, B.B., Booker Porter, T.K., Kinninger, L.A. and Trautmann, M.E. (2012) Clinical Relevance of Anti-Exenatide Antibodies: Safety, Efficacy and Cross-Reactivity with Long-Term Treatment. Diabetes, Obesity and Metabolism, 14, 546-554. http://dx.doi.org/10.1111/j.1463-1326.2012.01561.x 